\title{
Research on Enterprise Financial Customer Classification Method and Preference Based on Intelligent Algorithm
}

\author{
Ze Fu, ${ }^{1}$ Bo Zhang $\mathbb{D}^{2}{ }^{2}$ Lingjun Ou, ${ }^{3}$ Kaiyang Sun, ${ }^{4}$ Xinyi Sun, ${ }^{5}$ and Ningyan Chen ${ }^{6}$ \\ ${ }^{1}$ Yiwu Innovation Institute, Yiwu Industrial \& Commercial College, Yiwu 322000, China \\ ${ }^{2}$ School of Information and Communication Engineering, Communication University of China, Beijing 100024, China \\ ${ }^{3}$ Aston University, Birmingham B47ET, UK \\ ${ }^{4}$ Monash University, Melbourne, VIC 3800, Australia \\ ${ }^{5}$ Navigation College, Dalian Maritime University, Dalian 116026, China \\ ${ }^{6}$ South China Normal University, Zhaoqing 526000, China
}

Correspondence should be addressed to Bo Zhang; zhangbo2015@cuc.edu.cn

Received 22 September 2021; Accepted 11 November 2021; Published 28 November 2021

Academic Editor: Deepak Gupta

Copyright (c) $2021 \mathrm{Ze} \mathrm{Fu}$ et al. This is an open access article distributed under the Creative Commons Attribution License, which permits unrestricted use, distribution, and reproduction in any medium, provided the original work is properly cited.

\begin{abstract}
Compared with the past questionnaire survey, this paper applies the intelligent algorithm developed rapidly in recent years to identify the tendency of customers to buy financial products in the market. In addition, for the single state customer classification indicators based on the previous demographic information and action information, it is proposed to combine the action of market activities with demographic information; that is, the static integrated customer classification index is further combined with the improved neural network model to study the classification and preference of enterprise financial customers. Firstly, the enterprise financial customer classification model based on neural network algorithm is studied. Aiming at the shortcomings of easy falling into the local optimal solution of neural network algorithm, slow convergence speed of algorithm, and difficult setting of network structure, combined with the characteristics of genetic algorithm, the concept of adaptive genetic neural network algorithm is proposed. Then, the design of adaptive genetic neural network model is studied. Secondly, combined with the customer data of a financial enterprise and the characteristics of enterprise finance, this paper analyzes the risk influencing factors of enterprise financial customers, analyzes the customer data, evaluates the enterprise financial customers through the adaptive genetic neural network model, and realizes the classification of enterprise financial customers. Through an example, it is proved that the enterprise financial customer classification and preference model based on the adaptive genetic neural network algorithm discussed in this paper has better customer classification accuracy and can provide better method support for enterprise financial customer management.
\end{abstract}

\section{Introduction}

Customers in modern society have more choices. Customers' needs have been personalized. The competition among enterprises will also become fierce [1]. At the same time, with the progress of production technology, the differences between commodities become smaller, and the focus of competition changes from "product-centered" to "customer-centered" [2]. Only the enterprises that meet the needs of customers as quickly as possible can realize the sales of products. Only financial enterprises that can quickly respond to the individuation and change of customers can survive and develop in the fierce mar- ket competition. The most important index of enterprise management has changed from profit and cost to customer satisfaction. Customer relationship management is an important means for financial enterprises to gain competitive advantage. Therefore, this paper uses the improved self-adaptive genetic neural network customer classification model, which can distinguish the customers with different purchasing tendencies of financial enterprises and provide financial enterprises with different customers as the target, differentiated, and effective marketing services, which not only reduces the cost but also improves the operating profit and facilitates the financial customer relationship management of enterprises [3]. 
The effective classification of customers is the key to maintain and manage customer relationships in various fields, so it has attracted much attention. This paper puts forward a new method of customer value classification based on BP-AdaBoost, which is based on BP and AdaBoost algorithm, and analyzes the characteristics of different customers according to the designed evaluation system. AdaBoost algorithm is used to form a strong classifier, so as to improve the accuracy of traditional BP network [4]. Experiments show that this method is also effective for the application of financial customer classification, and the accuracy is satisfactory.

By collecting 2000 social media reviews of financial banks on the Internet, we studied the research framework for establishing financial bank data sets. In today's intelligent network, the role of social media in various fields cannot be ignored, including financial institutions. It can provide ways and channels to analyze needs and understand how to improve product quality and service, so that financial institutions can tailor personalized needs for customers. This framework uses various machine learning methods and technologies to classify emotions [5]. With fierce market competition, many financial institutions have to take sales as the first premise and use the quickest way to focus on "possible" customers. At the same time of competition, although financial institutions have a series of documents and methods to control risks, the borrower's intention is not obvious, which leads to a great reduction in the controllability of risks. In view of this serious phenomenon, we study the method of machine learning to promote handwriting analysis, which reflects a certain degree of intention from the unique properties of handwriting, and the recognized handwriting association is helpful to map individuals to corresponding personality types. When using machine learning technology to collect the samples of personal management questionnaire, it shows that extroversion is related to financial behavior as a "risk seeker" [6]. The early warning model of Internet finance is of great significance for enterprises in the financial field to prevent and control risks. We propose an improved K-means algorithm based on quantum evolution, which combines initial value and risk value to determine the risk early warning interval, and introduce quantitative algorithm into this algorithm to improve the search efficiency, so as to get the accurate early warning interval. Finally, we calculate the risk value with GMDH prediction mining method. Comparing nearly 10,000 data of Internet financial companies with real financial institutions, it shows that its model is available and effective [7].

\section{Adaptive Genetic Neural Network Algorithm}

2.1. Theory of Artificial Neural Networks. Artificial neural network is a complex system composed of many processing units similar to biological neurons [8].

2.1.1. Neuron Model. Artificial neural network is composed of artificial neurons as many basic processing units [9]. To establish an artificial neural network, we must first decide the artificial neuron model. Figure 1 is a general model structure diagram of artificial neurons.

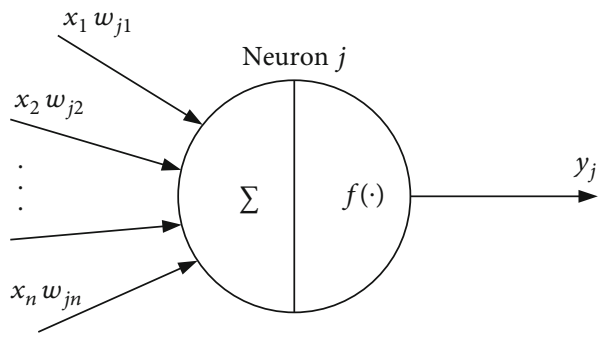

Figure 1: Neuron model.

2.1.2. BP Algorithm. BP algorithm is the most commonly used algorithm in neural network model learning. BP algorithm is a supervised learning algorithm. The idea of $\mathrm{BP}$ algorithm is that initial weights and thresholds are first provided to the network; an actual output value of the network is calculated from the input value $[10,11]$; then, the desired output value is compared with the actual output value; such learning is repeated on the training samples based on the obtained weights and thresholds of the error correction network, and finally, the error between the actual output and the desired output is minimized.

The specific process is as follows:

It is assumed that the neural network is an $I$ input unit and a $K$ output unit, the implicit layer is a layer, and the $J$ unit is shared. The BP algorithm is executed primarily to minimize the square error between the actual output, and the expected output obtained after the data is input into the network. The formula for the sum of squared errors is as follows.

$$
E=\frac{1}{2} \sum_{k=1}^{k}\left(d_{k}-o_{k}\right)^{2}
$$

Among them, $o_{k}=f\left(\right.$ net $\left._{k}\right)$ is the actual output value of neuron $k$ in the output layer, $d_{k}$ is the expected output value of neuron $k$ in the output layer, and $y_{j}$ is the output value of hidden layer neuron $j$.

For the $E$ value, in order to achieve the goal of the ideal value, it is necessary to change the weight value of the network. First, adjust the connection weights between the implicit layer and the output layer.

$$
w_{k j}(t+1)=w_{k j}(t)+\Delta w_{k j}
$$

In the above formula, the value obtained by the gradient method is the adjusted value of the connection weight between the implicit layer and the output layer.

$$
\Delta w_{k j}=-\eta \frac{\partial E}{\partial w_{k j}}=\eta\left(d_{k}-o_{k}\right) f^{\prime}\left(\operatorname{net}_{k}\right) y_{j} .
$$

In the above formula, $\eta$ is the normal value, which is expressed as the iteration step.

In a similar manner, you can adjust the join weights between the input layer and the implicit layer. Formula 
adjustment is as follows:

$$
v_{j i}(t+1)=v_{j i}(t)+\Delta v_{j i} .
$$

In the above formula, $\Delta v_{j i}$ is the adjustment amount for determining the connection weight between the input layer and the implicit layer by the gradient method. It can be obtained from the following formula:

$$
\Delta v_{j i}=-\eta \frac{\partial E}{\partial v_{j i}}=\eta \sum_{k=1}^{k}\left(d_{k}-o_{k}\right) f^{\prime}\left(\text { net }_{k}\right) w_{k j} f^{\prime}\left(\text { net }_{j}\right) x_{i} .
$$

When there are samples, if there are $P$ training samples, the total error sum form of the above calculation method is as follows:

$$
E_{p}=\frac{1}{2} \sum_{p=1}^{P} \sum_{k=1}^{K}\left(d_{k}-o_{k}\right)^{2}
$$

As long as the operation is repeated for $P$ samples as described above, $E_{p}$ reaches the minimum requested value, and the algorithm ends.

2.2. Adaptive Genetic Algorithm. Adaptive genetic algorithm is evolved from basic genetic algorithm $[12,13]$. It has strong global search performance and strong local search ability and accelerates the convergence speed to a certain extent. But it also has inherent defects.

2.2.1. Improvement of Adaptive Genetic Algorithm. The adaptive change formulas of crossover probability $P_{c}$ and mutation probability $P_{m}$ used in traditional adaptive genetic algorithm are as follows:

$$
\begin{aligned}
& p_{c}= \begin{cases}\frac{k_{1}\left(f_{\max }-f^{\prime}\right)}{f_{\max }-f_{\mathrm{avg}}}, & f^{\prime} \geq f_{\mathrm{avg}}, \\
k_{2}, & f^{\prime}<f_{\mathrm{avg}}\end{cases} \\
& p_{m}= \begin{cases}\frac{k_{3}\left(f_{\max }-f\right)}{f_{\max }-f_{\mathrm{avg}}}, & f \geq f_{\mathrm{avg}} . \\
k_{4}, & f<f_{\mathrm{avg}}\end{cases}
\end{aligned}
$$

Among them, $f_{\text {avg }}$ is the average fitness value of all individuals in the population, $f_{\max }$ is the maximum individual fitness value in the population, $f^{\prime}$ is the fitness value of the individual with the larger fitness value among the two individuals to be crossed, and $f$ is the fitness value of the individual to be mutated, any number comes from $k 1, k 2, k 3, k 4,0$ and 1.

Srinivas et al., by proposing $K 1=K 2=K, K 3=K 4=K^{\prime}$, represent adaptive adjustment curves for crossover probabilities and change probabilities in Figures 2 and 3.

As shown in the figure, in the early stage of evolution, individuals with higher fitness values may not be the global optimal solution.

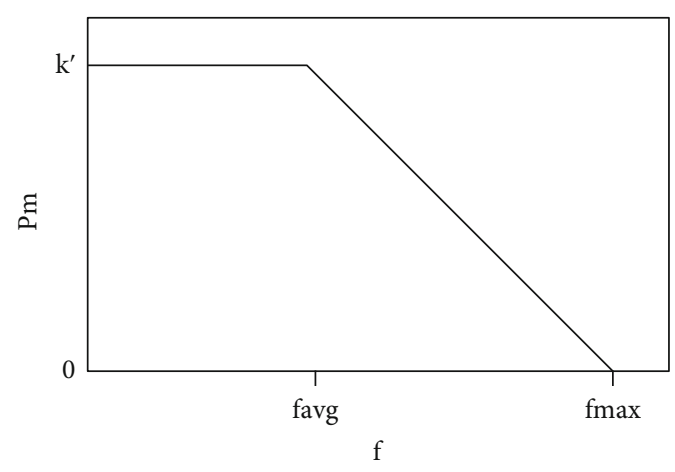

FIgURE 2: Variation curve of mutation probability.

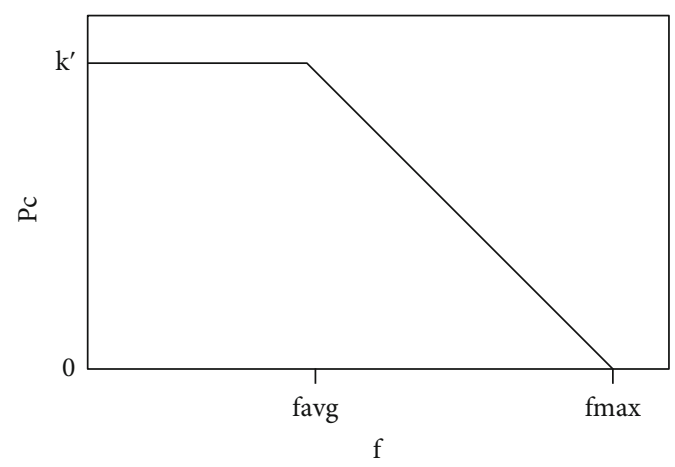

FIgURE 3: Cross probability change curve.

To solve this problem, this paper proposes an adaptive genetic algorithm. In addition, in order to maintain a better individual pattern after the evolution of the algorithm, the adaptive adjustment curve in $f_{\max }$ should be smoothed as much as possible. The formulas of crossover probability and mutation probability are as follows.

$p_{c}= \begin{cases}p_{c 1} * \frac{1}{\exp \left(\left(f^{\prime}-f_{\mathrm{avg}}\right) /\left(f_{\max }-f_{\mathrm{avg}}\right)\right)}, & f^{\prime} \geq f_{\mathrm{avg}}, \\ p_{c 1}, & f^{\prime}<f_{\mathrm{avg}}\end{cases}$

$p_{m}= \begin{cases}p_{m 1} * \frac{1}{\exp \left(\left(f-f_{\mathrm{avg}}\right) /\left(f_{\max }-f_{\mathrm{avg}}\right)\right)}, & f \geq f_{\mathrm{avg}}, \\ p_{m 1}, & f<f_{\mathrm{avg}}\end{cases}$

where $f^{\prime}$ is the fitness value of the larger of the two individuals to be crossed, $f_{\text {avg }}$ is the average fitness value of all individuals in the population, $f_{\text {max }}$ is the largest individual fitness value in the population, $f$ is the fitness value of the variant individual, and $P_{c 1}$ and $P_{m 1}$ are the largest crossover probability and mutation probability, respectively.

The adaptive adjustment curves of crossover probability and mutation probability are shown in Figures 4 and 5. 


\subsection{Adaptive Genetic Algorithm Evolutionary Neural Network}

2.3.1. Defects of Neural Networks. BP neural network is the most widely used model of neural network in classification $[14,15]$, and this algorithm is simply analyzed here. BP algorithm is an effective algorithm, but it also has some defects in practical application:

(1) Once a complex problem is solved, the result will fall into a local minimum, which will lead to the failure of learning

(2) If the learning speed decreases, the convergence speed will definitely slow down, but if it increases, the correction will not only be excessive but also cause vibration and divergence

(3) The number of input and output nodes of the network can be known according to the problem, and the selection of the number of hidden nodes is based on experience and lacks theoretical guidance

(4) The robustness is poor, and the initial setting value of the network has great influence on the overall performance of the network

Because BP neural network still has a series of defects [16, 17], we need to make some improvements to optimize its performance in order to get more satisfactory results. In this paper, the improved adaptive genetic algorithm is combined with neural network to make up for the abovementioned one or two shortcomings of neural network and the shortcomings of genetic algorithm itself, such as the reduction of convergence efficiency in the early stage and the middle and late stage.

2.3.2. Adaptive Genetic Neural Network. Up to now, the combination direction of genetic algorithm and neural network can be roughly divided into the following three types $[18,19]$.

(1) Optimizing network connection weights and thresholds

(2) Optimizing the topological structure of the network

(3) Optimize the learning rules of the network

The first optimization method is used most for BP neural network. This process corresponds to dividing the whole network into two steps. In the first step, genetic algorithm is used to derive the initial weights and thresholds of the network. In the second step, BP algorithm is applied to finally complete the training of the network. There are three key aspects in the optimization process: the expression of chromosome, the definition of fitness function, and the construction of genetic operator.

(1) Chromosome expression (i.e., coding mode)

Real numbers are used here. Because the scope of network rights is unclear, using real numbers can avoid coding becoming difficult. In addition, the efficiency of the learning

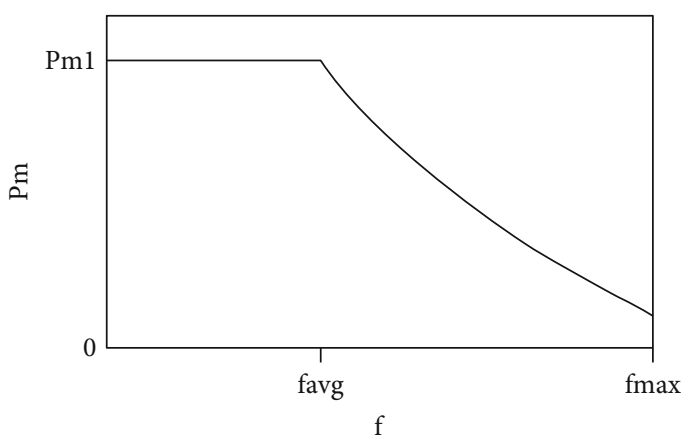

FIgURE 4: Variation curve of mutation probability after improvement.

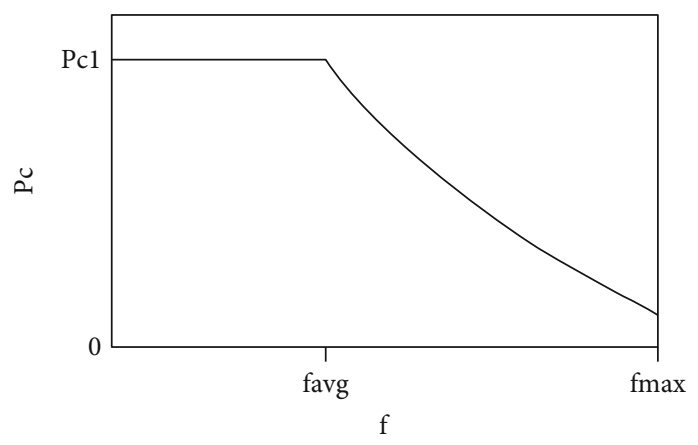

Figure 5: Modified curve of crossover probability.

algorithm can be improved without coding and coding. Real numbers are easy to retrieve in a large space, which can meet the accuracy requirements of genetic algorithm [20].

Taking a three-layer neural network as an example, chromosome length is as follows:

$$
l=W_{1}+b_{1}+W_{2}+b_{2}
$$

Chromosomes are composed of $l$ random numbers between 0 and 1 .

(2) Definition of fitness function

After determining the initial weights and thresholds of the BP neural network, an estimate of the number of inputs for training can be used as an evaluation function of the chromosomes. The evaluation function may be expressed as the reciprocal of the absolute value of the error between the actual output value and the expected output value obtained by the adaptation function, namely,

$$
f=\frac{1}{\sum_{i=1}^{n} \sum_{j=1}^{k}\left|y_{i j}-o_{i j}\right|} .
$$

\section{(3) Construction of selection operator}

By using the best individual preservation strategy, the individual with the highest adaptability in the current group 
is prevented from participating in the subsequent crossmeaning and mutation operations. By replacing the individual with the lowest adaptability in the generation group, the best individual obtained so far will not be destroyed, and the convergence of the algorithm can be guaranteed.

(4) Construction of crossover operator and mutation operator

Adaptive crossover operators and mutation operators change adaptively according to the evolution of groups [21, 22].

For BP neural network which determines the overall structure, the algorithm flow is as follows.

(1) Group Initialization. $N$ groups of initial network weights are randomly generated, and each group is used as a chromosome

(2) Select the Operation. Calculate the proportion corresponding to the fitness value of each chromosome, and select $N$ individuals to the next generation by roulette and the best individual preservation strategy

(3) Cross operation

(4) Mutation Operation. The number of iteration steps reached is (5), otherwise (2)

(5) Substituting the best chromosome into the initial value and threshold value of BP neural network and continuing in the training group until the error accuracy, or the set maximum algebra is reached

The algorithm flow chart is shown in Figure 6:

\section{Research on Classification and Preference of Enterprise Financial Customers Based on Adaptive Genetic Neural Network Algorithm}

3.1. Parameter Modification of BP Neural Network. In this paper, the gradient descent method is mainly used to study the classification and preference of enterprise financial customers by using BP neural network, and the weight of each BP neuron in the network is adjusted [23]. The specific operation process is as follows.

Analyze the output of hidden nodes in the network

$$
y_{i}=f\left(\sum_{j} \omega_{i j} x_{j}-\theta_{i}\right)=f\left(\text { net }_{i}\right) .
$$

Among them:

$$
\text { net }_{i}=\sum_{j} \omega_{i j} x_{j}-\theta_{i}
$$

In the three-layer BP neural network structure, the input of output nodes is the output of hidden nodes.

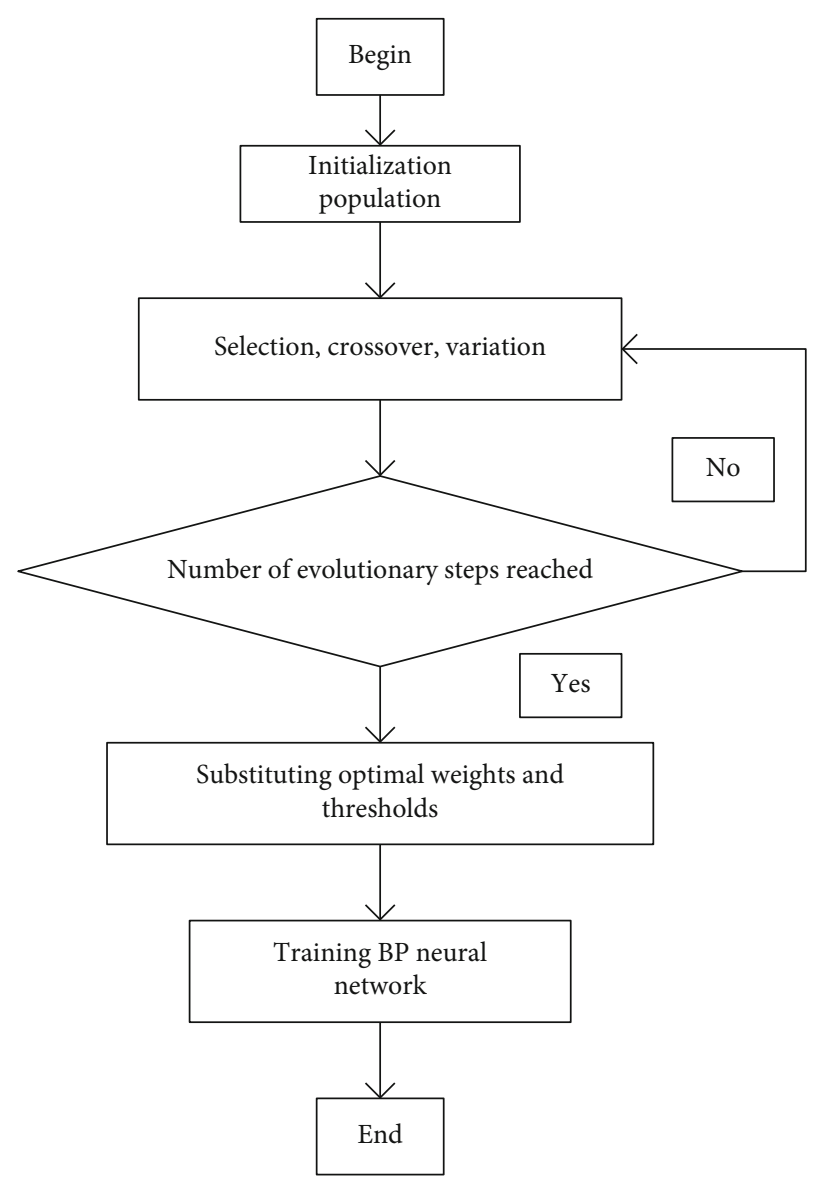

FIGURE 6: Flow chart of adaptive genetic neural network algorithm.

TABLE 1: Sample index numeralization.

\begin{tabular}{lcccccc}
\hline Indicators & Default & Housing & Loan & Marital & Contact & Day \\
\hline 0 & No & No & No & - & - & - \\
1 & Yes & Yes & Yes & Married & Unknown & $0-9$ \\
2 & - & - & - & Divorced & Telephone & $10-$ \\
& & & & & & 19 \\
3 & - & - & - & Single & Cellular & $20-$ \\
& & & & & & 31
\end{tabular}

$$
\begin{gathered}
s_{k}=f\left(\sum_{i} u_{i j} y_{i}-\theta_{k}\right)=f\left(\text { net }_{k}\right), \\
\operatorname{net}_{k}=\sum_{i} u_{i j} y_{i}-\theta_{k} .
\end{gathered}
$$

The error between the output node of BP neural network and the actual value of training samples is calculated as follows.

$$
E=\frac{1}{2} \sum_{k}\left(z_{k}-s_{k}\right)^{2}=\frac{1}{2} \sum_{k}\left(z_{k}-f\left(\sum_{i} u_{i j} f\left(\sum_{j} \omega_{i j} x_{j}-\theta_{i}\right)-\theta_{k}\right)\right)^{2} .
$$


TABLe 2: Sample index digitization (continued).

\begin{tabular}{lccccc}
\hline Indicators & Education & Balance & Month & Poutcome & Plays \\
\hline 1 & Unknown & 0以下 & $1-3$ & Unknown & -1 \\
2 & Secondary & $0-999$ & $4-6$ & Other & $0-99$ \\
3 & Primary & $1000-4999$ & $7-9$ & Failure & $100-199$ \\
4 & Tertiary & More than 5000 & $10-12$ & Success & $200-299$ \\
5 & - & - & - & - & More than 300 \\
\hline
\end{tabular}

TABle 3: Sample index digitization (continued).

\begin{tabular}{lccc}
\hline Indicators & Age & Duration & Job \\
\hline 1 & $0-19$ & $0-99$ & Admin \\
2 & $20-29$ & $100-299$ & Unknown \\
3 & $30-39$ & $300-499$ & Unemployed \\
4 & $40-49$ & $500-599$ & Management \\
5 & $50-59$ & $600-899$ & Housemaid \\
6 & 60 以上 & $900-999$ & Entrepreneur \\
7 & - & $1000-1699$ & Student \\
8 & - & More than 1700 & Self-employed \\
9 & - & - & Blue-collar \\
10 & - & - & Retired \\
11 & - & - & Technician \\
12 & - & - & Services \\
\hline
\end{tabular}

The weights between output nodes, hidden nodes, and errors are analyzed. If the output nodes in BP neural network are independent of each other, the following relations exist.

$$
\frac{\partial E}{\partial U_{i j}}=-\delta_{t} y_{i}
$$

3.2. Sample Data Acquisition. The experimental data comes from the enterprise financial customer data set in UCI machine learning database. This data is about whether the marketing products of financial enterprises can finally be purchased by customers. A total of 2000 sets of data samples were taken. Each sample consists of 16 attributes and 1 category. The 16 attributes can be divided into three categories.

Demographic variables: describe some basic information of customers.

Demographic variables: describe some basic information of customers, including age (age), work (job), marital status (marriage), education, default, annual average balance, housing, and personal loan (loan).

Some variables of communication between financial enterprises and customers about current marketing activities include communication mode (contact), the date of the last communication in a month, month of the last communication in a certain year, the last communication time (duration), and the number of communications with this customer during this activity.

Some variables that a financial enterprise communicates with a customer about the last event include the last event and the last communication from the customer (pdays), the number of communications with the customer prior to the event (previous), and the result of the last marketing event (poutcome).

This category indicates whether the customer purchased the marketing products of the financial enterprise this time.

3.3. Sample Data Preprocessing. The steps of data preprocessing are as follows. Firstly, the nonnumerical attributes in the sample are numerized to discretize the continuous attributes. Then, the description and statistics of sample indicators are carried out to analyze the rationality of the application model. After that, principal component analysis is used to extract the hidden main features as input variables of neural network.

3.3.1. Numerical Properties. The nonnumerical attributes in the index are numerized, and the continuous attributes are discrete. The specific process is shown in Tables 1,2 , and 3 , and the attributes are listed as the actual attribute values.

3.3.2. Descriptive Statistics of Sample Indicators. In order to explain whether the model is feasible, first of all, the sample indicators are explained and counted, and the statistical results are expressed in Table 4.

From the various values shown by the deviation and peak coefficients in Table 4, these coefficients of age, balance, and month are close to zero, that is, close to normal distribution, but other indicators hardly follow. Therefore, the traditional statistical model, which is constrained by many indexes, cannot be used to analyze and solve this problem. However, because these constraints cannot limit the model based on neural network technology, the classification and preference model based on genetic neural network can be applied here.

3.3.3. Sample Impact Factor Analysis. Factor analysis is a statistical technique, which can summarize a large number of indicators into a few factors and explain a large number of observed facts. When the main features are fuzzy and covered by redundant data, it is difficult for general neural networks to identify them $[24,25]$. However, if the main features are clear and the same accuracy is obtained, the operation efficiency of the network model is greatly improved.

Principal component analysis is carried out on the numerical attribute data, and the eigenvalues and contribution rates of the correlation coefficient arrangement of variables are shown in Table 5 . 
TABle 4: Sample index description statistics.

\begin{tabular}{|c|c|c|c|c|c|c|c|}
\hline Variable & $N$ & Minimum value & Maximum value & Mean value & Standard deviation & Skewness & Kurtosis \\
\hline Age & 2000 & 1 & 6 & 3.65 & 1.021 & 334 & -0.540 \\
\hline Job & 2000 & 1 & 12 & 7.06 & 3.493 & -0.24 & -1.181 \\
\hline Marital & 2000 & 1 & 3 & 1.67 & 0.879 & 0.695 & -1.346 \\
\hline Education & 2000 & 1 & 4 & 2.70 & 0.947 & 0.301 & -1.319 \\
\hline Default & 2000 & 0 & 1 & 0.02 & 0.133 & 0.256 & 50.704 \\
\hline Balance & 2000 & 1 & 4 & 2.32 & 0.725 & 0.534 & 0.174 \\
\hline Housing & 2000 & 0 & 1 & 0.57 & 0.495 & -0.275 & -1.926 \\
\hline Loan & 2000 & 0 & 1 & 0.16 & 0.365 & 1.877 & 1.524 \\
\hline Contact & 2000 & 1 & 3 & 2.38 & 0.892 & -0.823 & -1.235 \\
\hline Day & 2000 & 1 & 3 & 2.06 & 0.789 & -0.115 & -1.384 \\
\hline Month & 2000 & 1 & 4 & 2.42 & 0.809 & 0.290 & -0.395 \\
\hline Duration & 2000 & 1 & 8 & 2.34 & 1.380 & 1.758 & 3.244 \\
\hline Campaign & 2000 & 1 & 32 & 2.72 & 2.840 & 4.069 & 24.843 \\
\hline Pdays & 2000 & 1 & 5 & 1.47 & 1.096 & 2.267 & 3.874 \\
\hline Previous & 2000 & 0 & 20 & 0.54 & 1.607 & 5.191 & 38.656 \\
\hline Poutcome & 2000 & 1 & 4 & 1.36 & 0.797 & 2.038 & 2.757 \\
\hline
\end{tabular}

TABLE 5: Sample variance contribution rate table.

\begin{tabular}{lccccccc}
\hline \multirow{2}{*}{ Composition } & \multicolumn{3}{c}{ Initial eigenvalue } & \multicolumn{2}{c}{ Extract sum of squares and load } & Rotation sum of square loading \\
& Total & Variance\% & Cumulative\% & Total & Variance\% & Cumulative\% & Total \\
\hline 1 & 2.544 & 15.898 & 15.898 & 2.544 & 15.898 & 15.898 & 2.506 \\
2 & 1.569 & 9.808 & 25.706 & 1.569 & 9.808 & 25.706 & 1.475 \\
3 & 1.414 & 8.835 & 34.541 & 1.414 & 8.835 & 34.541 & 1.408 \\
4 & 1.242 & 7.761 & 42.302 & 1.242 & 7.761 & 42.302 & 1.251 \\
5 & 1.159 & 7.246 & 49.548 & 1.159 & 7.246 & 49.548 & 1.256 \\
6 & 1.100 & 6.877 & 56.425 & 1.100 & 6.877 & 56.425 & 1.254 \\
7 & 1.023 & 6.396 & 62.820 & 1.023 & 6.396 & 62.820 & \\
8 & 0.937 & 5.858 & 68.678 & & & & 1.063 \\
9 & 0.880 & 5.499 & 74.176 & & & & \\
10 & 0.837 & 5.232 & 79.409 & & & \\
11 & 0.803 & 5.018 & 84.427 & & & \\
12 & 0.753 & 4.706 & 89.133 & & & & \\
13 & 0.620 & 3.877 & 93.010 & & & & \\
14 & 0.555 & 3.471 & 96.482 & & & & \\
15 & 0.397 & 2.483 & 98.965 & & & & \\
16 & 0.166 & 1.035 & 100.000 & & & & \\
\hline
\end{tabular}

It can be seen from Table 5 that the correlation coefficient matrix of variables has seven largest characteristic roots, which are $2.554,1.599,1.414,1.242,1.159,1.100$, and 1.023, respectively. The cumulative contribution rate of eigenvalue reaches $62.80 \%$; that is to say, it contains most of the information of the original index. Based on the principle that the eigenvalue is greater than 1, seven main components can be extracted. In order to show the influence degree of principal components on the original index, it is necessary to establish their original factor load matrix. In order to facilitate the extraction of information and maximize the dispersion of principal components, the orthogonal rotation method is used to further process the structurally simplified rotated factor load matrix, and the results are shown in Table 6. Finally, using these seven main component factors, instead of the original indicators, we can study the classification and preference of customers.

Table 6 only shows the load above 0.4 , which clearly indicates the economic significance of each major component.

After determining the economic significance of the main component factors and calculating the score coefficient of the sample factors, the linear combination equation of 7 main component indexes and the original 16 indexes can 
TABLE 6: Factor load matrix after sample rotation.

\begin{tabular}{|c|c|c|c|c|c|c|c|}
\hline \multirow{2}{*}{ Attribute } & \multicolumn{7}{|c|}{ Composition } \\
\hline & 1 & 2 & 3 & 4 & 5 & 6 & 7 \\
\hline Pdays & 0.923 & & & & & & \\
\hline Poutcome & 0.913 & & & & & & \\
\hline Previous & 0.813 & & & & & & \\
\hline Housing & & -0.727 & & & & & \\
\hline Contact & & 0.687 & & & & & \\
\hline Month & & 0.584 & & & & & \\
\hline Marital & & & 0.836 & & & & \\
\hline Age & & & -0.800 & & & & \\
\hline Balance & & & & -0.656 & & & \\
\hline Default & & & & 0.635 & & & \\
\hline Loan & & & & 0.591 & & & \\
\hline Job & & & & & -0.806 & & \\
\hline Education & & & & & 0.742 & & \\
\hline Day & & & & & & 0.744 & \\
\hline Campaign & & & & & & 0.693 & \\
\hline Duration & & & & & & & 0.914 \\
\hline
\end{tabular}

TABle 7: Prediction errors corresponding to different hidden layer node numbers.

\begin{tabular}{lccc}
\hline $\begin{array}{l}\text { Number of hidden } \\
\text { layer nodes }\end{array}$ & $\begin{array}{c}\text { Prediction } \\
\text { error }\end{array}$ & $\begin{array}{c}\text { Number of hidden } \\
\text { layer nodes }\end{array}$ & $\begin{array}{c}\text { Prediction } \\
\text { error }\end{array}$ \\
\hline 4 & 0.1176 & 9 & 0.0186 \\
5 & 0.0933 & 10 & 0.0267 \\
6 & 0.0658 & 11 & 0.1064 \\
7 & 0.0872 & 12 & 0.0561 \\
8 & 0.0143 & 13 & 0.0751 \\
\hline
\end{tabular}

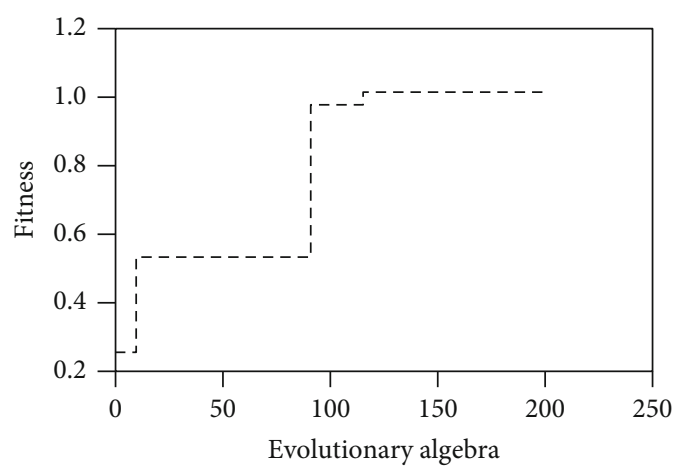

FIGURE 7: Variation diagram of fitness curve of traditional AGABP.

be obtained. These seven main component indicators can be replaced by the original 16 indicators as a new index system, which can be used as the input data of the follow-up model.

\section{Experiment}

4.1. Definition of Model Structure and Selection of Parameters. The sample data obtained from the principal

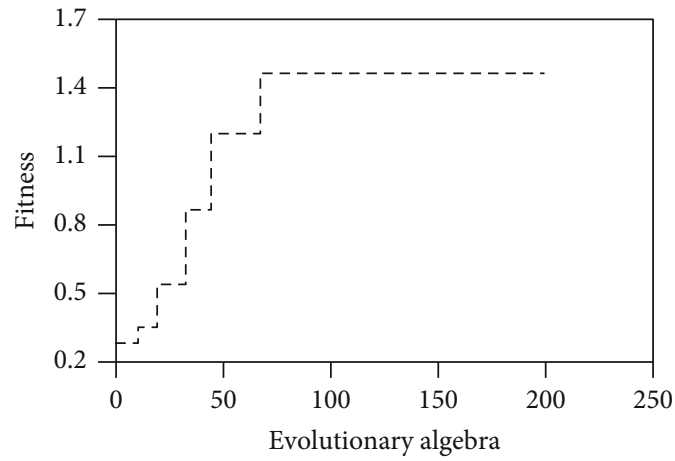

Figure 8: Change diagram of fitness curve of improved AGABP.

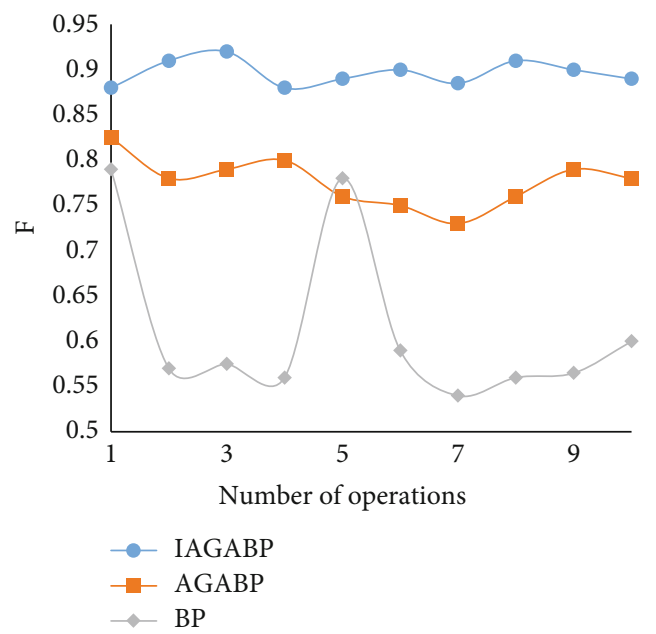

FIGURE 9: Comparison results of different algorithms.

component analysis of the original index of the sample is composed of 7 variables, so the number of input rows of the network is 7 .

Determine the number of output layer nodes. The number of output layer nodes is determined by the number of desired output results of the network. This model divides the customer's output into two types, 1 for the customer's purchase and 0 for the customer's nonpurchase. Therefore, the number of output nodes of BP network model should be 1 .

Determine the number of hidden layer nodes. Although there is no accepted standard so far, too much or too little is not appropriate. If the number is too large, the learning time will become longer, and the popularity of the network will decline. If the number is too small, the network may not be able to train or recognize new samples. Fault-tolerant performance is also greatly reduced. Here, the calculation is based on the following empirical equation (21).

$$
n=\sqrt{a+b}+c
$$

Use estimates and show the results in Table 7.

The data in the table shows that when the number of implicit layer nodes is 8 , the prediction error corresponding 


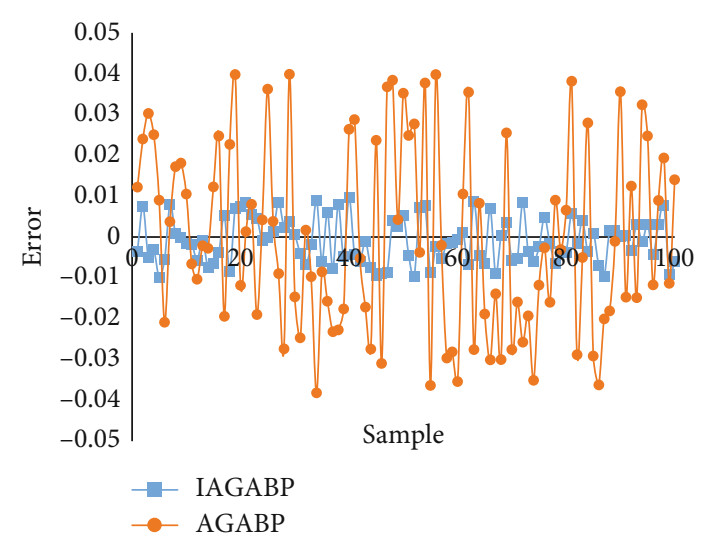

FIGURE 10: IAGABP and AGABP classification error result diagram of test set.

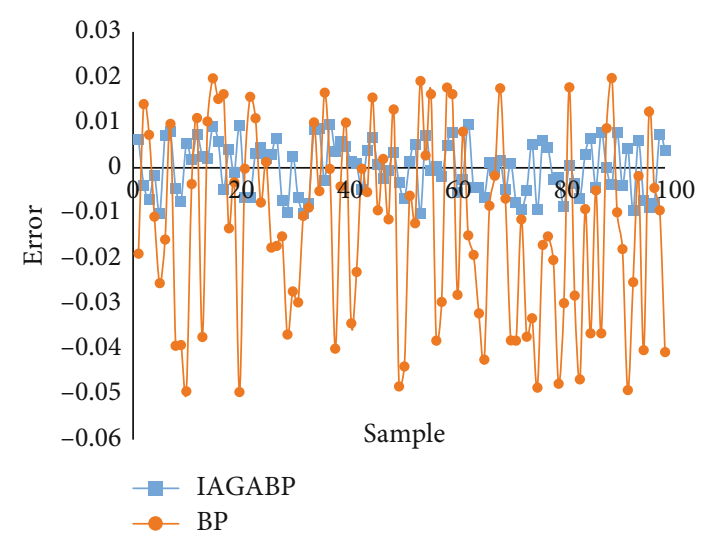

FIGURE 11: IAGABP and AGABP classification error result diagram of test set.

to BP neural network is the smallest, and 8 is selected as the number of implicit layer nodes. Make up a 7-8-1 network.

4.2. Network Training and Result Verification. In this paper, 2000 corporate financial customer data samples are randomly divided into two groups, and 1900 groups of data are used as training group training network. Another data set 100 examples detect the generalized performance of the network as a test set and predict customer categories and preferences.

\subsubsection{Algorithm Performance Analysis}

(1) Comparison of Convergence Rate of Genetic Algorithm. Set the end algebra to 200, and Figure 7 converges faster than Figure 8. This shows that the algebra of the latter should be much smaller than that of the former after setting a fixed convergence value, so the latter is much more efficient than the former.

People expect the same from all algorithms. I hope to converge early and realize overall optimization, but they are just a pair of contradictory requirements. As far as genetic algorithm is concerned, if it converges quickly, the
TABle 8: Summary of prediction effect of each model.

\begin{tabular}{lccc}
\hline $\begin{array}{l}\text { Neural } \\
\text { network type }\end{array}$ & $\begin{array}{c}\text { BP } \\
\text { neural } \\
\text { network } \\
\text { (BP) }\end{array}$ & $\begin{array}{c}\text { Traditional } \\
\text { adaptive genetic } \\
\text { neural network } \\
\text { (AGABP) }\end{array}$ & $\begin{array}{c}\text { Improved adaptive } \\
\text { genetic neural } \\
\text { network } \\
\text { (IAGABP) }\end{array}$ \\
\hline $\begin{array}{l}\text { Classification } \\
\text { accuracy }\end{array}$ & $73 \%$ & $82 \%$ & $97 \%$ \\
\hline
\end{tabular}

speed close to the best state will become faster, which cannot guarantee the diversity of combinations and makes it difficult to realize the overall optimization. In order to obtain the global optimum value, we must try our best to avoid falling into local extremum. This is because the population must keep the diversity of individuals as much as possible, so it cannot converge quickly. Therefore, if you want to combine the two, you should consider them comprehensively and choose from them. The method proposed in this paper not only pursues global optimization but also considers the operation speed of genetic algorithm, so as not to affect the global convergence speed as much as possible, thus finding a good balance between them.

(2) Stability Analysis. In order to verify the performance of the adaptive genetic neural network algorithm proposed in this paper, BP neural network algorithm, traditional adaptive genetic neural network algorithm, and improved adaptive genetic neural network algorithm are executed several times, respectively, and the correctness of the calculation method is evaluated according to $F$ evaluation index. Here, the higher the $F$ value, the higher the correctness of the result. The stability of the algorithm can be judged by whether the data has great changes. The experimental results are shown in Figure 9.

Through the above experimental results, it can be seen that the $F$ value of the improved adaptive genetic neural network algorithm proposed in this paper is obviously higher than the other two algorithms in the global range, and the fluctuation in the global range is small, reflecting better stability. Among them, BP neural network algorithm has the smallest $F$ value, which fluctuates greatly, and the lowest accuracy rate drops to less than $60 \%$. As far as the traditional adaptive genetic neural network algorithm is concerned, its stability and accuracy have been improved to some extent, but compared with the improved adaptive genetic neural network algorithm, there are still some shortcomings, and the latter maintains better stability and higher accuracy.

From the above experimental results, it can be seen that the $F$ value of the adaptive genetic neural network algorithm proposed in this paper is significantly higher than the other two algorithms in the global situation, and it fluctuates less in the global situation and shows better stability. The $F$ number of BP neural network algorithm is the smallest, and it is easy to change greatly, and the lowest accuracy will reach below $60 \%$. In the traditional adaptive genetic neural network algorithm, its stability and accuracy have been improved to some extent, but compared with the improved 
adaptive genetic neural network algorithm, it is not sufficient, and the latter maintains better stability and high accuracy.

4.2.2. Comparison of Classification Accuracy. Figures 10 and 11 show the error results of classification test for 100 sets of data. It can be seen intuitively that the error of IAGABP is closer to 0 than that of AGABP and BP. To sum up, it can be concluded that IAGABP is more accurate than BP and AGABP in the application of corporate financial customer classification and preference.

According to the prediction results, if the indication with error within 0.01 is classified accurately, the prediction effects of each model on samples are summarized as shown in Table 8 .

According to the data, the classification accuracy of adaptive genetic neural network is the highest, which is $24 \%$ and 15\% higher than BP neural network and traditional adaptive genetic neural network, respectively.

\section{Conclusion}

Through the above research, we should provide targeted services to different types of customers.

\section{(1) Buy customers}

Maintaining such customers plays a stable and important role in the income of financial enterprises. Therefore, financial enterprises must take measures to maintain long-term contact with them. For example, keep the communication channel smooth at all times. When issuing products or services, financial enterprises should let customers know in time, actively attract their opinions and actively improve to meet their needs. For example, the fee and annual fee of various cards are reduced, the agency business fees are eliminated, and the remittance fee rate and personal insurance rate are reduced.

\section{(2) Unpurchased customers}

For such low-value customers, there is almost no contribution rate to financial products, but there are small deposits. For their needs, financial enterprises can reduce their counters and use the electronic equipment of financial enterprises. Such customers basically have no development value. The development cost is much higher than the income they create for the enterprise. Therefore, there is no need to carry out any marketing of products and services for such customers.

After analyzing the classification and preference of existing customers, financial enterprises should strive to provide corresponding services for different types of customers, maintain existing high-value customers to the maximum extent without wasting resources, and obtain more benefits for corporate finance. The future work needs to be carried out from the following points: (1) The data dimension of financial customers is insufficient, so we hope to analyze and predict the data under a large amount of data and adopt a deep multilayer learning complex model for learning and analysis; (2) intelligent algorithms can be applied to financial credit classification to dig deep into risky customers.

\section{Data Availability}

The experimental data used to support the findings of this study are available from the corresponding author upon request.

\section{Conflicts of Interest}

The authors declared that they have no conflicts of interest regarding this work.

\section{Acknowledgments}

This work is supported by the Department of Education of Zhejiang Province.

\section{References}

[1] L. Zhang, T. Liu, Y. Xie, Z. Zeng, and J. Chen, "A new classification method of nanotechnology for design integration in biomaterials," Nanotechnology Reviews, vol. 9, no. 1, pp. 820$832,2020$.

[2] O. Senvar, D. Akburak, and N. Yel, "Customer oriented intelligent DSS based on two-phased clustering and integrated interval type-2 fuzzy AHP and hesitant fuzzy TOPSIS," Journal of Intelligent and Fuzzy Systems, vol. 39, no. 5, pp. 61216143, 2020.

[3] F. Pramaswari, A. P. Nasution, and S. L. Nasution, "The effect of branding quality and service quality on customer satisfaction through Financial Technology (FinTech) at PT. WOM Finance branch Rantauprapat," Budapest International Research and Critics Institute (BIRCI-Journal) Humanities and Social Sciences, vol. 4, no. 2, pp. 2995-3004, 2021.

[4] X. Shi, G. Li, K. Li, J. Liu, and R. Wang, "Customer classification method of logistics enterprises based on BP-AdaBoost," Journal of Physics Conference Series, vol. 1670, 2020.

[5] I. Raicu, "Financial banking dataset for supervised machine learning classification," Informatica Economica, vol. 23, pp. 37-49, 2019.

[6] S. Thomas, M. Goel, and D. Agrawal, "A framework for analyzing financial behavior using machine learning classification of personality through handwriting analysis," Journal of behavioral and experimental Finance, vol. 26, p. 100315, 2020.

[7] M. S. Dong, "Intelligent early warning of Internet financial risks based on mobile computing," International Journal of Mobile Computing and Multimedia Communications, vol. 11, no. 2, pp. 61-78, 2020.

[8] S. Chavlis and P. Poirazi, "Drawing inspiration from biological dendrites to empower artificial neural networks," Current Opinion in Neurobiology, vol. 70, pp. 1-10, 2021.

[9] C. M. Loo Christopher, T. Sasikumar, and T. Page, "Crack growth detection on Al/Sicp using acoustic monitoring and artificial neural network," Materials Today: Proceedings, vol. 16, no. P2, pp. 604-611, 2019.

[10] S. Park, S. Seo, C. Jeong, and J. Kim, "The weights initialization methodology of unsupervised neural networks to improve clustering stability," The Journal of Supercomputing, vol. 76, no. 8, pp. 6421-6437, 2020.

[11] M. V. Narkhede, P. P. Bartakke, and M. S. Sutaone, "A review on weight initialization strategies for neural networks," Artificial Intelligence Review, vol. 2, pp. 1-32, 2021. 
[12] S. Mirjalili, "Biogeography-based optimisation," in Evolutionary Algorithms and Neural Networks vol, pp. 57-72, Springer, 2019.

[13] M. Zhou, Y. Long, W. Zhang et al., "Adaptive genetic algorithm-aided neural network with channel state information tensor decomposition for indoor localization," IEEE Transactions on Evolutionary Computation, vol. 25, no. 5, pp. 913-927, 2021.

[14] V. Afzali Gorooh, S. Kalia, P. Nguyen et al., "Deep neural network cloud-type classification (DeepCTC) model and its application in evaluating PERSIANN-CCS," Remote Sensing, vol. 12, no. 2, p. 316, 2020.

[15] S. Xu, N. Feng, K. Liu, Y. Liang, and X. Liu, "A weighted fuzzy process neural network model and its application in mixedprocess signal classification," Expert Systems with Applications, vol. 172, no. 1, 2021.

[16] Y. Ma, L. Li, Z. Yin, A. Chai, M. Li, and Z. Bi, "Research and application of network status prediction based on BP neural network for intelligent production line," Procedia Computer Science, vol. 183, no. 20, pp. 189-196, 2021.

[17] D. Zhang, W. Li, X. Wu, and X. Lv, “Application of simulated annealing genetic algorithm optimized back propagation (BP) neural network in fault diagnosis," International Journal of Modeling Simulation \& Scientific Computing, vol. 10, no. 4, pp. 1950024-1950049, 2019.

[18] P. Mohamed Jebran and S. Gupta, "Pre-diabetic retinopathy identification using hybridGenetic algorithm-neural network classifier," Journal of Physics: Conference Series, vol. 1937, no. $1,2021$.

[19] X. Feng, J. Zhao, and E. Kita, "Genetic algorithm-based optimization of deep neural network ensemble," The Review of Socionetwork Strategies, vol. 15, no. 1, pp. 27-47, 2021.

[20] M. S. Kumar, "Image retrieval using heuristic approach and genetic algorithm," The Journal of Computer Information Systems, vol. 8, no. 4, pp. 1563-1571, 2019.

[21] H. J. Lim, S. H. Choi, J. Oh, B. S. Kim, S. Kim, and J. H. Yang, "Adaptive ground control system of multiple-UAV operators in a simulated environment," Aerospace Medicine and Human Performance, vol. 90, no. 10, pp. 841-850, 2019.

[22] S. Rani and B. Suri, "Investigating different metrics for evaluation and selection of mutation operators for Java," International Journal of Software Engineering and Knowledge Engineering, vol. 31, no. 3, pp. 311-336, 2021.

[23] J. Wang, Y. Wen, Y. Gou, Z. Ye, and H. Chen, "Fractionalorder gradient descent learning of BP neural networks with Caputo derivative," Neural networks, vol. 89, pp. 19-30, 2017.

[24] E. Moulton, K. Bradbury, M. Barton, and D. Fein, "Factor analysis of the childhood autism rating scale in a sample of two year olds with an autism spectrum disorder," Journal of Autism and Developmental Disorders, vol. 49, no. 7, pp. 2733-2746, 2019.

[25] L. Hou, Z. Zhang, Y. du, X. Wang, and C. Zhao, “An allergic risk factor analysis of extraordinary daytime only urinary frequency in children," Risk Management and Healthcare Policy, vol. 14, pp. 2683-2688, 2021. 